\title{
Testicular prosthesis: Patient satisfaction and sexual dysfunctions in testis cancer survivors
}

\author{
Francesco Catanzariti ${ }^{1}$, Benedetta Polito ${ }^{2}$, Massimo Polito ${ }^{1}$ \\ ${ }^{1}$ Polytechnic University of Marche, Faculty of Medicine, Department of Odontostomatologic and Specialized Clinical Sciences, \\ Urology Clinic, Department of General and Specialized Surgery, University Hospital, Ancona, Italy; \\ 2 Degree in Educational and childhood Psychology, Salesian Pontifical University, Rome, Italy.
}

\begin{abstract}
Summary Purpose: We studied patient satisfaction about sexual activity after prosthesis implantation using validated questionnaires with the aim to discover if testicular prosthesis could be responsible of sexual dysfunctions (erectile dysfunction or premature ejaculation). Materials and Methods: We evaluated a total of 67 men who underwent radical orchiectomy for testicular cancer and a silicon testicular prosthesis implantation from January 2008 to June 2014 at our Hospital. These patients completed 5 validated questionnaires the day before orchiectomy and 6 months after surgery: the International Index of Erectile Function 5 (IIEF5), the Premature Ejaculation Diagnostic Tool (PEDT), the Body Exposure during Sexual Activities Questionnaire (BESAQ), the Body-Esteem Scale and the Rosenberg SelfEsteem Scale. We also evaluated 6 months after surgery any defects of the prosthesis complained by the patients.

Results: The questionnaires completed by patients didn't show statistically significant changes for erectile dysfunction ( $p>$ $0.05)$ and premature ejaculation $(p>0.05)$. On the contrary the psychological questionnaires showed statistically significant change for the BESAQ $(p<0.001)$ and the Body Esteem Scale $(p<0.001)$, but not for the Rosenberg Self-Esteem Scale ( $p>0,05)$. A total of 15 patients $(22.37 \%)$ were dissatisfied about the prosthesis: the most frequent complaint (8 patients; $11.94 \%)$ was that the prosthesis was firmer than the normal testis.

Conclusions: Testicular prosthesis implantation is a safe surgical procedure that should be always proposed before orchiectomy for cancer of the testis. The defects complained by patients with testicular prosthesis are few, they don't influence sexual activity and they aren't able to cause erectile dysfunction or premature ejaculation.
\end{abstract}

KEY WORDS: Testicular cancer; Testicular prostheses; Sexual dysfunction; Patient satisfaction.

Submitted 26 March 2016; Accepted 5 August 2016

\section{INTRODUCTION}

Testicular cancer is one of the most frequent carcinoma in young males. In USA about 8000 men discover to be affected by testicular cancer every year and about 400 men of these die for this disease in a year, but fortunately the five-year survival rate of patients with testicular cancer is $95 \%$ (1). Peak incidence is in the third decade of life for non-seminoma, and in the fourth decade for pure seminoma (2), so that this disease occurs during the most important period of life for sexual activity. The find of being affected by cancer of the testis and the following loss of the testis from the scrotal sac after orchiectomy are both responsible of a great psychological trauma in these young men, as described in literature (3). The implantation of testicular prostheses could be the solution, but even if good aesthetic results can be obtained, the presence of an artificial testis can be cause of shame and loss of self confidence during sexual activity.

Most of the papers in literature analyse patient satisfaction for testicular prosthesis just about aesthetic side (size, texture, weight, position), in our study, on the contrary, we studied patient satisfaction after prosthesis implantation about sexual activity using validated questionnaires with the aim to discover if testicular prosthesis could be responsible of any sexual dysfunction (erectile dysfunction or premature ejaculation).

\section{Methods}

In lieu of a formal ethics committee, the principles of the Helsinki Declaration were followed. A total of 95 men underwent radical orchiectomy for testicular cancer from January 2008 to June 2014 at our Hospital. Of these patients, 67 underwent also a silicon testicular prosthesis implantation.

This group of patient completed 5 validated questionnaires the day before orchiectomy and 6 months after surgery: the International Index of Erectile Function - 5 (IIEF-5) (4) to evaluate erectile function; the Premature Ejaculation Diagnostic Tool (PEDT) (5) to assess premature ejaculation; the Body Exposure during Sexual Activities Questionnaire (BESAQ) (6) a 28 item scale to measure anxiety during sexual activity and to evaluate desires and attempts to selectively avoid exposing one's body (or parts of one's body) to sexual partners; the Body-Esteem Scale (7) and the Rosenberg Self-Esteem Scale (8) those are respectively 35 and 10 item scales created to measure self confidence of each patient towards sexuality and other general aspects of life.

We also evaluated any defects of the prosthesis complained by the patients within 6 months after surgery: pain, abnormal size of the prosthesis (larger or smaller than the normal testis), prosthesis texture (firmer than the normal testis), sense of coldness of the prosthesis and 
abnormal position of the prosthesis in the scrotal sac (higher than the normal testis).

\section{Surgical technique}

We used a silicone testicular prosthesis, with a protective suture guard for easy implantation. The size of the prosthesis to use was determined by ultrasound before surgery. All the implants were placed at the initial surgery via the inguinal approach and none was inserted after orchiectomy as a second procedure. The prosthesis was fixed in the scrotum with a non-absorbable suture.

\section{Statistical methods}

All the scores of questionnaires before and after surgery and defects of the prosthesis complained by the patients were collected into a database. General descriptive statistics were measured for categorical and continuous variables. We performed Student's t-test to compare mean score of all the five questionnaires (IIEF5, PEDT, BESAQ, Body-Esteem Scale and Rosenberg Self-Esteem Scale) before and after surgery. Student's t-test was considered significant if $<0.05$. Statistical analyses were performed with IBM SPSS Statistics Base ${ }^{\circledR}$.

\section{RESULTS}

From January 2008 to June 2014 we performed 95 orchiectomy for cancer of the testis and 67 (70.52\%) patients of these decided also to undergo a silicone testicular prosthesis implantation. The remaining 28 (29.47\%) patients refused testicular prosthesis for different reasons (Table 1): 15 (53.57\%) patients were afraid of infection and of the need of a second surgery to repair it; 10 (35.71\%) patients didn't care of remaining just with one testis in the scrotal sac; 3 (10.71\%) patients didn't want prosthesis because they couldn't accept an artificial testis in the scrotal sac for psychological reasons (shame with the partner during sexual activity or bad memories evoked by self-palpation of the testicular prosthesis). Age

Table 1.

Reasons of refusal of testicular prosthesis implantation.

\begin{tabular}{|lc|}
\hline Fear of infection & No. (\%) \\
\hline Regardless of remaining with only one testicle & $15(53.57 \%)$ \\
\hline Psychological reasons (shame with the partner or other) & $10(35.71 \%)$ \\
\hline
\end{tabular}

Table 2.

Age and marital status of patients who accepted testicular prosthesis implantation.

\begin{tabular}{|lc|}
\hline Mean age & 34.39 \\
\hline Median age & 33.00 \\
\hline Standard deviation of age & 11.24 \\
\hline $\begin{array}{l}\text { No. marital status at surgery (\%): } \\
\quad \text { Single or divorced }\end{array}$ & $45(67.16)$ \\
$\quad$ Married or a partner in a steady relationship & $22(32.83)$ \\
\hline $\begin{array}{l}\text { No. marital status 6 months later (\%): } \\
\text { Single or divorced } \\
\quad \text { Married or a partner in a steady relationship }\end{array}$ & $48(71.64)$ \\
\hline
\end{tabular}

and marital status of patients who accepted testicular prosthesis implantation are described in Table 2.

Between the 67 patients who underwent implantation two (2.98\%) had inflammation with scrotal edema that healed in few days with anti-inflammatory and just one (1.49\%) had an hematoma resorbed spontaneously without a second surgery. No major complications (extrusion or migration of the prosthesis) have been described (Table 3).

The questionnaires (Table 4) completed by patients before and 6 months after surgery (mean follow up: 49.42 months) didn't show statistically significant changes in the score for erectile dysfunction ( $p>0.05)$ and premature ejaculation $(\mathrm{p}>0.05)$. On the contrary the psychological questionnaires showed statistically significant change in the score for the BESAQ $(p<0.001)$ and the Body Esteem Scale $(p<0.001)$, but not for the Rosenberg Self-Esteem Scale ( $p>0.05)$. We also collected data about dissatisfaction of patients about the prosthesis and defects of the prosthesis complained by the patients within 6 months from surgery (Table 5).

Fifteen patients (22.37\%) were dissatisfied about the prosthesis: one $(1.49 \%)$ patient reported chronic pain, six (8.95\%) men reported abnormal size of the prosthesis compared to the normal testis (five patients reported that the prosthesis was larger than the normal testis and one patient reported that the prosthesis was smaller than the normal testis); eight (11.94\%) patients reported that the prosthesis was firmer than the normal testis; two (2.98\%) patients complained of sense of coldness of the prosthesis and four $(5.97 \%)$ patients reported that the prosthesis was higher than the normal testis in the scrotal sac.

\section{Discussion}

Our study is one of the few studies available in the literature about testicular prosthesis satisfaction after orchiecto-

\section{Table 3.}

Complications of testicular prosthesis implantation.

\begin{tabular}{|lc|}
\hline Inflammation with scrotal edema & No. (\%) \\
\hline Hematoma & $2(2.98 \%)$ \\
\hline
\end{tabular}

Table 4.

Score changes in the 5 questionnaires (IIEF-5, PEDT, BESAQ, Body Esteem Scale and Rosenberg Self Esteem Scale) at testicular prosthesis implantation $\left(t^{\circ}\right)$ and 6 months later $\left(t^{1}\right)$.

\begin{tabular}{llcc}
\hline & & Mean score \pm SD & p value change \\
\hline IIEF-5 & $t^{0}$ & $22.83 \pm 2.46$ & \\
& $t^{1}$ & $22.20 \pm 2.84$ & $p>0.05$ \\
\hline PEDT & $t^{0}$ & $7.79 \pm 5.30$ & \\
& $t^{1}$ & $8.39 \pm 5.27$ & $p>0.05$ \\
\hline BESAQ & $t^{0}$ & $37.59 \pm 11.5$ & \\
& $t^{1}$ & $46.17 \pm 10.91$ & $p<0.001$ \\
\hline Body Esteem Scale & $t^{0}$ & $85.14 \pm 12.14$ & \\
& $t^{1}$ & $77.77 \pm 10.24$ & $p<0.001$ \\
\hline Rosenberg Self Esteem Scale & $t^{0}$ & $27.30 \pm 3.04$ & \\
& $t^{1}$ & $26.45 \pm 3.26$ & $p>0.05$
\end{tabular}


Table 5.

Dissatisfaction of patients about the prosthesis and defects of the prosthesis complained by the patients 6 months after surgery.

\begin{tabular}{|lc|}
\hline & No. (\%) \\
\hline Dissatisfaction of patients about the prosthesis & $15(22.37)$ \\
\hline Defects of the prosthesis complained by the patients & \\
\hline Chronic pain & $1(1.49)$ \\
\hline $\begin{array}{l}\text { Abnormal size of the testicular prosthesis } \\
\text { Larger than the normal testis } \\
\text { Smaller than the normal testis }\end{array}$ & $5(7.46)$ \\
\hline Testicular prosthesis firmer than the normal testis & $1(1.49)$ \\
\hline $\begin{array}{l}\text { Sense of coldness of the prosthesis } \\
\text { Testicular prosthesis higher than the normal testis } \\
\text { in the scrotal sac }\end{array}$ & $2(11.94)$ \\
\hline
\end{tabular}

my for testicular cancer. This is a great and serious lack in andrology if we consider that there is a great number of studies in literature about breast implants satisfaction after mastectomy. Moreover most of these few studies $(9,10)$ analyse just satisfaction about aesthetic factors (size, texture, weight, position) but not about sexual activity after testicular prosthesis implantation. Furthermore the few studies $(11,12)$ who analyse this aspect use simple and generic questions without using validated questionnaires. The only paper in literature analysing sexual activity after testicular prosthesis implantation with validated questionnaires is the study by Turek et al. (13). These authors used the same psychological validated questionnaires (BESAQ, Body-Esteem Scale and Rosenberg Self-Esteem) that we used in our study but they didn't use IEEF-5 and PEDT for the analysis of erectile dysfunction and premature ejaculation before and after testicular prosthesis implantation as we did. Moreover their study is about testicular prosthesis implantation not only after orchiectomy for cancer of the testis but also for torsion, trauma or agenesis of the didimus so that Turek et al. included in their work 73 children and 76 adults.

Our study demonstrated that testicular prosthesis implantation is a safe surgical procedure with few risks of complications as the other papers cited showed. The dissatisfaction rate is quite low (22.37\%). The changes of the mean scores of psychological questionnaires are statistically significant for BESAQ and Body Esteem Scale $(p<0.001)$ but not for the Rosenberg Esteem Scale ( $p>0.05)$ and the changes of the mean scores of IIEF-5 and PEDT before and 6 months after orchiectomy are not statistically significant. These results demonstrate that a testicular prosthesis implantation modifies psychological approach towards the partners during sexual activity because some patients with testicular prosthesis feel shame of it and they try to not show the prosthesis during sexual activity. We also showed that testicular prosthesis doesn't cause sexual dysfunction (erectile dysfunction or premature ejaculation). However our study has some limitations. First the follow up after testicular prosthesis implantation is short (6 months), second we didn't compare sexual activity satisfaction of our patients with sexual activity satisfaction of patients those refused testicular prosthesis implantation.

\section{Conclusions}

This study confirms that testicular prosthesis implantation is a safe surgical procedure that should be always proposed before orchiectomy for cancer of the testis because complications and defects complained by patients with testicular prosthesis are few.

We also showed for the first time that implant doesn't influence sexual activity and it isn't able to cause erectile dysfunction or premature ejaculation even if further studies with a control group are needed to confirm this.

\section{REFERENCES}

1. Cancer Facts \& Figures 2014 and the NCI Surveillance Epidemiology and End Results (SEER) database 1975-2010.

2. Bosl GJ, Motzer RJ.Testicular germ-cell cancer. N Engl J Med. 1997; 337:242.

3. Skoogh J, Steineck G, Cavallin-Ståhl E, et al. Feelings of loss and uneasiness or shame after removal of a testicle by orchidectomy: a population-based long-term follow-up of testicular cancer survivors. Int J Androl. 2011; 34:183-92.

4. Rosen RC, Cappelleri JC, Smith MD, et al. Development and evaluation of an abridged, 5-item version of the International Index of Erectile Function (IIEF-5) as a diagnostic tool for erectile dysfunction. Int J of Imp Res. 1999; 11:319.

5. Symonds T, Perelman MA, Althof S, et al. Development and validation of a premature ejaculation diagnostic tool. Eur Urol. 2007; 52:565.

6. Cash TF, Maikkula CL, Yamamiya Y. Baring the body in the bedroom: Body image, sexual self-schemas, and sexual functioning among college women and men. Electronic Journal of Human Sexuality 2004, http://www.ejhs.org/volume7/bodyimage.html

7. Franzoi SL, Shields SA. The Body-Esteem Scale. Multidimensional structure and sex differences in a college population. J of Pers Assess. 1984; $48: 173$.

8. Rosenberg M. Society and the adolescent self-image. Princeton, NJ 1965; Princeton University Press.

9. Adshead J, Khoubehi B, Wood J, Rustin G. Testicular implants and patient satisfaction: a questionnaire-based study of men after orchidectomy for testicular cancer. BJU Int. 2001; 88:559-62.

10. Xylinas E, Martinache G, Azancot V, et al. Testicular implants, patient's and partner's satisfaction: a questionnaire-based study of men after orchidectomy. Prog Urol. 2008; 18:1082-6.

11. Incrocci L, Bosch JL, Slob AK. Testicular prostheses: body image and sexual functioning. BJU Int. 1999; 84:1043-5.

12. Yossepowitch O, Aviv D, Wainchwaig L, Baniel J. Testicular Prostheses for Testis Cancer Survivors: Patient Perspectives and Predictors of Long-Term Satisfaction. J Urol. 2011; 186:2249-2252.

13. Turek PJ, Master VA. Testicular Prosthesis Study Group. Safety and effectiveness of a new saline filled testicular prosthesis. J Urol. 2004; 172:1427-30.

\section{Correspondence}

Francesco Catanzariti, MD

frenzis83@gmail.com

Massimo Polito, MD

Polytechnic University of Marche, Faculty of Medicine,

Department of Odontostomatologic and Specialized Clinical Sciences, Urology Clinic, Department of General and Specialized Surgery,University Hospital, Ancona, Italy

Benedetta Polito, MD

Educational and Chlidhood Psychologist, Salesian Pontifical University, Rome, Italy 